\title{
Endoscopic Ultrasound Features of Multiple Endocrine Neoplasia Type 1-Related Versus Sporadic Pancreatic Neuroendocrine Tumors: A Single-Center Retrospective Study
}

\author{
Gianluca Tamagno \\ Mater Misericordiae University Hospital, gianlucatamagno@tiscali.it \\ Vanessa Scherer \\ University Hospital, Philipp's University \\ Alberto Caimo \\ Technological University Dublin, alberto.caimo@tudublin.ie
}

See next page for additional authors

Follow this and additional works at: https://arrow.tudublin.ie/scschmatart

Part of the Biostatistics Commons, and the Medicine and Health Sciences Commons

\section{Recommended Citation}

Tamagno, G., Scherer, V., Caimo, A., Bergmann, S. R., \& Kann, P. H. (2018). Endoscopic Ultrasound Features of Multiple Endocrine Neoplasia Type 1-Related versus Sporadic Pancreatic Neuroendocrine Tumors: A Single-Center Retrospective Study. Digestion, 98(2), 112-118. doi:10.1159/000487939

This Article is brought to you for free and open access by the School of Mathematics at ARROW@TU Dublin. It has been accepted for inclusion in Articles by an authorized administrator of ARROW@TU Dublin. For more information, please contact arrow.admin@tudublin.ie, aisling.coyne@tudublin.ie,gerard.connolly@tudublin.ie.

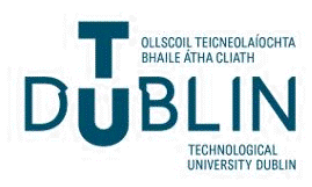




\section{Authors}

Gianluca Tamagno, Vanessa Scherer, Alberto Caimo, Simona Bergmann, and Peter Kann

This article is available at ARROW@TU Dublin: https://arrow.tudublin.ie/scschmatart/243 


\section{Endoscopic Ultrasound Features of Multiple Endocrine Neoplasia Type 1-Related versus Sporadic Pancreatic Neuroendocrine Tumors: A Single-Center Retrospective Study}

\author{
Gianluca Tamagno ${ }^{a, c}$ Vanessa Scherer ${ }^{a}$ Alberto Caimo $^{b}$ \\ Simona R. Bergmann ${ }^{\text {a }}$ Peter H. Kann ${ }^{a}$ \\ a Division of Endocrinology and Diabetology, University Hospital, Philipp's University, Marburg, Germany; ${ }^{\text {b School }}$ \\ of Mathematical Sciences, Dublin Institute of Technology, Dublin, Ireland; ' Department of Endocrinology/Diabetes \\ Mellitus, Mater Misericordiae University Hospital, Dublin, Ireland
}

CC Free Author
Copy - for per-
sonal use only

ANY DISTRIBUTION OF THIS ARTICLE WITHOUT WRITTEN CONSENT FROM S. KARGER AG, BASEL IS A VIOLATION OF THE COPYRIGHT.

Written permission to distribute the PDF will be granted against payment of a permission fee, which is based on the number of accesses required. Please contact permission@karger.com

\section{Keywords}

Pancreas - Neuroendocrine tumor - Multiple endocrine neoplasia type $1 \cdot$ Endoscopic ultrasound

\begin{abstract}
Aim: Pancreatic neuroendocrine tumors (pNETs) can occur in patients with a familial syndrome either as multiple endocrine neoplasia type 1 (MEN-1) or as sporadic tumors. Endoscopic ultrasound (EUS) has become one of the first-line investigations for PNET characterization. The ultrasonographic features of pNETs may differ depending on the familial versus sporadic pathogenesis of the tumor. Therefore, the EUS findings could help and direct the definition of a PNET with an impact on the most appropriate diagnostic and therapeutic patient management. Methods: In this single-center retrospective study, we reviewed the EUS features of 94 pNETs from 37 MEN-1 patients and 15 pNETs from 11 spo-
\end{abstract}

G.T. and V.S. equal contribution.

\section{KARGER}

๑) 2018 S. Karger AG, Basel

E-Mail karger@karger.com

www.karger.com/dig radic disease patients at the time of their first EUS assessment. We analyzed the most relevant morphological and ultrasonographic characteristics of the tumors and compared the findings between the 2 patient groups. Results: Patients with MEN-1 more likely present with multiple pNETs than patients with sporadic disease. Sporadic pNETs are usually much bigger than those due to MEN-1. Moreover, pNETs are more heterogeneous in patients with sporadic disease than in those with MEN-1. No statistical difference with regard to definition of the margins, morphology, and vascularization of the pNETs appears between the 2 groups. Conclusions: Patients with sporadic disease usually present with bigger and more heterogeneous pNETs than patients with MEN-1, who tend to present with a higher number of lesions. EUS can facilitate the precise characterization of a PNET, and the ultrasonographic features of the lesion can help and distinguish MEN-1-related versus sporadic disease.

(c) 2018 S. Karger AG, Basel

Dr. Gianluca Tamagno

Department of Endocrinology/Diabetes Mellitus

Mater Misericordiae University Hospital

Eccles Street, Dublin 7 (Ireland)

E-Mail gianlucatamagno@ tiscali.it

Prof. Peter H. Kann

Division of Endocrinology and Diabetology, Department of Gastroenterology Endocrinology and Metabolism, University Hospital of Marburg, Philipp's University Baldingerstrasse, DE-35043 Marburg (Germany)

E-Mail kannp@med.uni-marburg.de 


\section{Introduction}

Neuroendocrine tumors represent a heterogeneous group of neoplasms from a biological, anatomical, and clinical point of view. Approximately, 5\% of all neuroendocrine tumors originate from the pancreas and the estimated incidence of pancreatic neuroendocrine tumors (pNETs) is $0.32 / 100,000 /$ year [1]. From a pathogenetic perspective, pNETs can occur in patients with a familial syndrome or they can be sporadic as well [2]. Among the familial syndromes potentially leading to the development of a pNET, multiple endocrine neoplasia type 1 (MEN-1) is the definitely most frequent condition, followed by von Hippel-Lindau disease, neurofibromatosis 1 (von Recklinghausen disease), and the tuberous sclerosis complex [3]. The genetic background of the pNET determines some differences in the diagnostic and therapeutic approach and dictate specifically targeted followup plans. In such a setting, all investigations and techniques that can help with the determination of the familial rather than sporadic nature of the pNET are valuable.

In MEN- 1 patients, pNETs occur in $30-80 \%$ of the cases and may either secrete or not secrete hormones [4]. Moreover, the age of pNET onset is earlier in MEN-1 patients than in the patients with sporadic disease [5]. Though recommendations and guidelines have been published, the optimal managements of pNETs in MEN1 still remain controversial, especially with regard to the nonfunctioning tumors $[4,6,7]$.

In the last decade, endoscopic ultrasound (EUS) has become one of the first-line imaging investigations for the characterization of pNETs $[8,9]$ and has a clearly defined role in the multidisciplinary diagnostic approach to pNET [10]. In experienced hands, EUS has sensitivity for pNETs ranging between 80 and $90 \%$ and specificity above $95 \%$ $[11,12]$. In MEN-1 patients, EUS appears to be the most sensitive method for the detection of pNETs even when they are pretty small in size $[4,13-15]$. The diagnostic value of EUS can go beyond this aspect, as such a technique may help with the retrieval of histological or cytological specimens for pathology examination. Moreover, the EUS features of a pNET might differ depending on the familial versus sporadic nature of the tumor and can direct the subsequent steps of tumor diagnosis and localization. Therefore, the EUS findings by themselves might help and direct the accuracy of the definition of a pNET at the time of its first detection, with a possible impact on the most appropriate diagnostic and therapeutic management of the patient found to carry the lesion.
In our study, we have collected, analyzed, and compared the EUS features of pNETs occurring in a singlecenter series of patients with MEN-1 versus those of pNETs occurring sporadically.

\section{Methods}

In this single-center retrospective study, we have collected, reviewed, and analyzed the EUS characteristics of 94 pNETs from 37 patients with MEN-1 and 15 pNETs from 11 patients with sporadic disease at the time of their first EUS assessment (Table 1). The patients consecutively attended our institution over a 5-year time period (from September 2002 to July 2007).

EUS was carried out by 2 investigators using a Pentax FG-32UA and FG-36UX endosonoscope (Pentax Corporation, Tokyo, Japan) with a longitudinal $7.5 \mathrm{MHz}$ sector array in combination with a Hitachi EUB-420 or Hitachi EUB-525 computational system (Hitachi Medical Corporation, Tokyo, Japan) [16]. The vascularization of the tumors was assessed with duplex ultrasonography after a careful optimization of the Doppler technique and an appropriate gain setting for avoiding the appearance of noise.

Patients underwent EUS with conscious sedation. All EUS procedures were carried out as per the appropriate diagnostic management of the patients and were uneventful with regard to the occurrence of adverse events. With the goal of defining the EUS features of MEN-1-related versus sporadic pNETs, we have reviewed the most relevant morphological and ultrasonographic aspects of the tumors as described in the EUS reports and compared the findings between the 2 patient groups. First of all, the number and the size of the tumors have been recorded. Then, the definition of the margin (well defined versus not well defined), the morphology (round/oval versus speckled), the appearance (homogeneous versus heterogeneous), and the vascularization (hypervascular versus non-hypervascular) of the tumors have been evaluated.

The relevant data have been subsequently anonymized and stored in a password-protected system. The statistical evaluation of the data has been carried out using the analysis of variance when assessing the pNET maximum diameter, taking a $p$ value $<0.05$ as the cutoff for statistical significance. The EUS characteristics of the pNETs, including the number of lesions detected in each patient, have been analyzed using the Barnard's test, taking again a $p$ value $<0.05$ as the cutoff for significance. Barnard's test is an exact unconditional nonparametric test for the analysis of contingency tables, which uses a more computationally intensive algorithm for creating a rejection region [17], finally representing a more powerful alternative to Fisher's exact test.

This retrospective study has been carried out in accordance with the principles of the Declaration of Helsinki.

\section{Results}

Our study shows that, at the time of the first EUS detection of a pNET, patients with MEN-1 appear more likely to present with multiple lesions in comparison to 
Table 1. Clinicopathological features of 48 patients with one or more pNETs occurring in the setting of MEN-1 or as sporadic disease

\begin{tabular}{|c|c|c|c|c|}
\hline Patient & Age & Number of tumors & Maximum diameter, $\mathrm{mm}$ & Clinicopathological characterization \\
\hline \multicolumn{5}{|c|}{ Patients with MEN-1 } \\
\hline 1 & 32 & 6 & 21 & Nonfunctioning \\
\hline 3 & 77 & 1 & 10 & Nonfunctioning \\
\hline 4 & 35 & 2 & 10 & Nonfunctioning \\
\hline 5 & 38 & 4 & 5 & Nonfunctioning \\
\hline 8 & 45 & 2 & 12 & Nonfunctioning \\
\hline 9 & 25 & 2 & 6 & Nonfunctioning \\
\hline 10 & 52 & 2 & 6 & Insulinoma, nonfunctioning \\
\hline 11 & 18 & 1 & 4 & Insulinoma \\
\hline 12 & 39 & 2 & 6 & Nonfunctioning \\
\hline 17 & 58 & 2 & 26 & Insulinoma, nonfunctioning \\
\hline 18 & 65 & 5 & 12 & Nonfunctioning \\
\hline 19 & 33 & 2 & 11 & Nonfunctioning \\
\hline 20 & 29 & 5 & 26 & Insulinoma, nonfunctioning \\
\hline 21 & 50 & 2 & 13 & Nonfunctioning \\
\hline 22 & 46 & 1 & 5 & Nonfunctioning \\
\hline 23 & 28 & 2 & 4 & Nonfunctioning \\
\hline 24 & 48 & 6 & 12 & Nonfunctioning \\
\hline 25 & 38 & 1 & 5 & Gastrinoma \\
\hline 26 & 47 & 2 & 10 & Gastrinoma, nonfunctioning \\
\hline 27 & 38 & 1 & 13 & Nonfunctioning \\
\hline 28 & 52 & 2 & 25 & Gastrinoma \\
\hline 37 & 56 & 1 & 6 & Nonfunctioning \\
\hline \multicolumn{5}{|c|}{ Patients with sporadic disease } \\
\hline 1 & 63 & 1 & 8 & Nonfunctioning \\
\hline 2 & 68 & 1 & 60 & Nonfunctioning \\
\hline 3 & 63 & 2 & 6 & Nonfunctioning \\
\hline 4 & 59 & 3 & 24 & Nonfunctioning \\
\hline 5 & 26 & 1 & 65 & Nonfunctioning \\
\hline 6 & 29 & 2 & 45 & Insulinoma \\
\hline 7 & 69 & 1 & 7 & Glucagonoma \\
\hline 8 & 66 & 1 & 14 & Nonfunctioning \\
\hline 9 & 77 & 1 & 25 & Nonfunctioning \\
\hline 10 & 38 & 1 & 9 & Nonfunctioning \\
\hline 11 & 68 & 1 & 26 & Nonfunctioning \\
\hline
\end{tabular}


patients with the sporadic disease (2.5 vs. 1.4 tumors/ patient). Despite a pretty well-defined trend, such finding did not reach statistical significance $(p=0.163)$. On the contrary, pNETs occurring in patients with sporadic disease tend to be significantly bigger than those arising in the setting of MEN-1 $(p>0.001)$ and, more in the specific, the sporadic pNETs are 3 times bigger than the MEN-1-related lesions (23 vs. $7 \mathrm{~mm}$; Fig. 1). With regard to the tumor size, patients with sporadic disease also display a much greater variability than patients with MEN-1, presenting with lesions, which range from 4 to $65 \mathrm{~mm}$ versus 3 to $26 \mathrm{~mm}$. In sporadic disease patients, about half of the lesions had a maximum diameter between 10 and $30 \mathrm{~mm}$ and about a quarter of the lesions had a much bigger size, with maximum diameter above $40 \mathrm{~mm}$. In patients with MEN-1, two thirds of the pNETs had a diameter smaller than $10 \mathrm{~mm}$, with a non-negligible portion of them being just at the size limit for detection. Only very few lesions had a maximum diameter above $20 \mathrm{~mm}$ and none of them was close to $30 \mathrm{~mm}$ in size.

In patients with sporadic disease, the tumors appear to be significantly more heterogeneous than in patients with MEN-1 (Table 2). The large majority of MEN-1-related pNETs have a homogeneous appearance. An illustrative EUS picture of homogenous versus heterogeneous pNET is provided (Fig. 2a, b). On the contrary, in terms of the features concerning their EUS appearance, there is no statistical difference with regard to the definition of the margins, the morphology, and the vascularization of the pNETs between the 2 groups. However, the observation that 3 quarters of pNETs arising in MEN-1 patients have well-defined margins is remarkable, also considering the fact that the rate between lesions with well-defined versus not well-defined margins in sporadic disease patients is much more equilibrated. However, such observation did not achieve any statistical significance in our patient population. Finally, it may be worth noticing that almost all pNETs have a round/oval shape and appear to be hypervascular regardless of their pathogenesis.

\section{Discussion}

The diagnosis of pNETs is affected by a number of relevant challenges, which include the pathogenetic nature, the localization, the definition of the number of the tumors, and first of all their identification. These issues probably arise in a more striking way when the

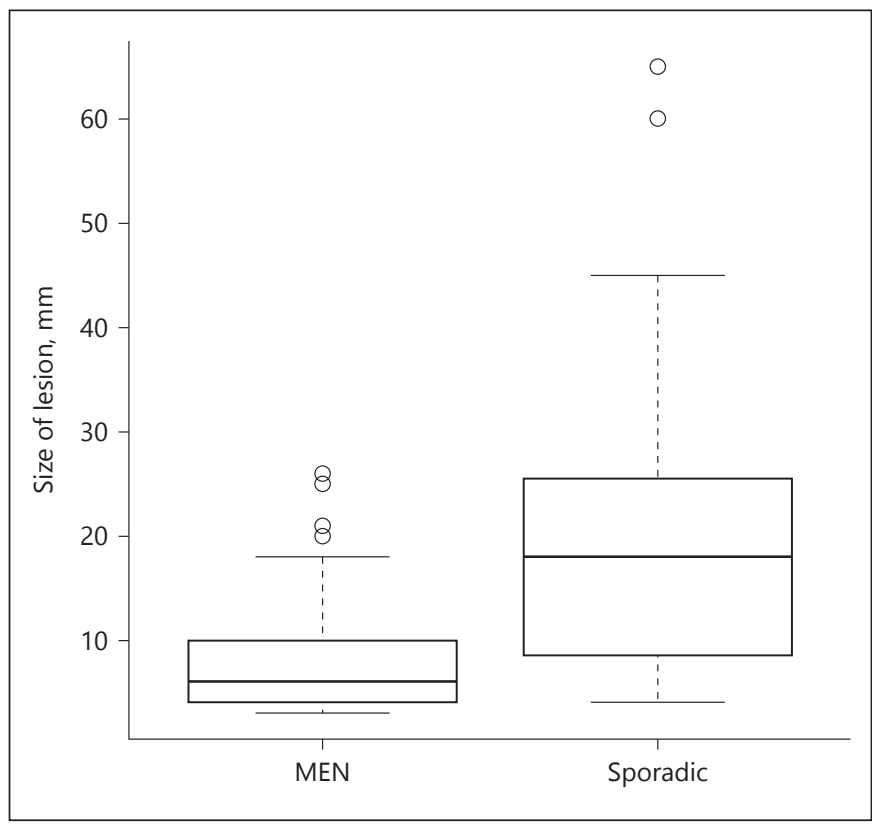

Fig. 1. Comparison of the size of pNETs detected by EUS in patients with MEN-1 (MEN) and in patients with sporadic disease (Sporadic). $p$ value $<0.001$

Table 2. Analysis of the most relevant EUS features of pNETs in patients with MEN-1 (MEN) and in patients with sporadic disease (Sporadic).

\begin{tabular}{lrrl}
\hline & MEN & Sporadic & $\begin{array}{l}\text { Barnard's test, } \\
p \text { value }\end{array}$ \\
\hline Well defined & 75 & 8 & 0.058 \\
Not well defined & 19 & 7 & 0.383 \\
\hline Round/oval & 92 & 14 & $<0.001$ \\
Speckled & 2 & 1 & \\
\hline Homogeneous & 74 & 3 & 0.525 \\
Heterogeneous & 20 & 12 & \\
\hline Non-hypervascular & 4 & 0 & \\
Hypervascular & 90 & 15 & \\
\hline
\end{tabular}

pNET does not secrete any hormone leading to a specific endocrine syndrome. In such a challenging setting, many biochemical markers or radiological techniques can be of value and should be used with the goal of clarifying in the most precise way the disease, as this point has an extremely important role for the establishment of the best plan of surgical and/or medical treatment and follow-up $[4,8,9]$. Moreover, in the case of 

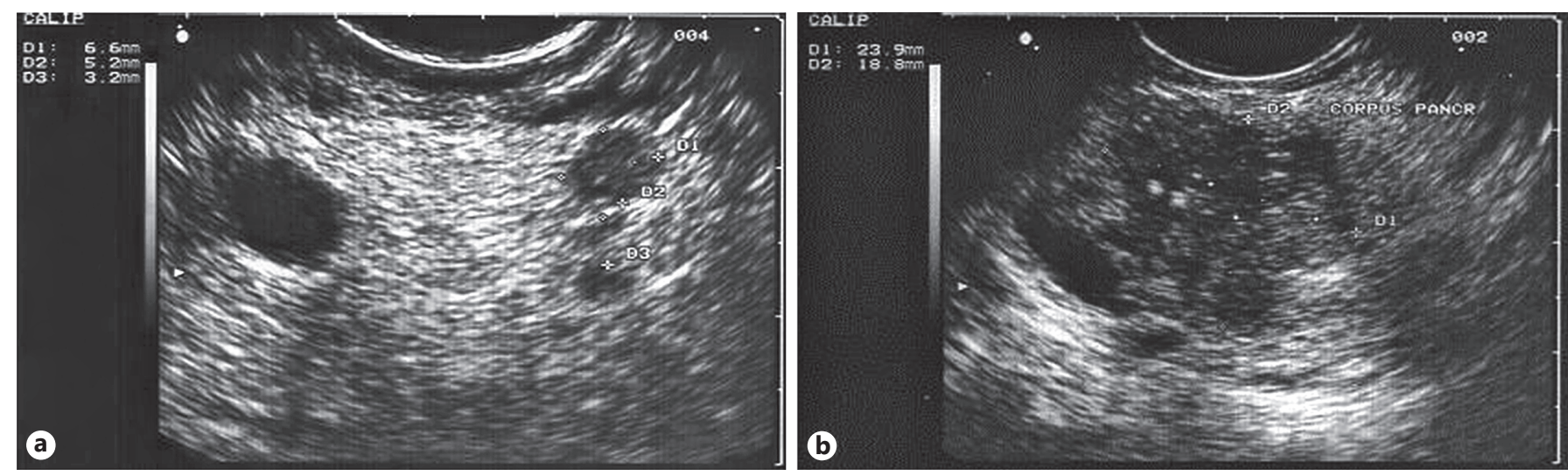

Fig. 2. An illustrative EUS picture of a homogeneous pNET (a) of a patient with MEN-1 and a heterogeneous pNET of a patient with sporadic disease $(\mathbf{b})$.

pNETs occurring in patients with a familial syndrome, like MEN-1, an early diagnosis is not only necessary for treating the patients on time but also extremely useful for defining the times of the successive follow-up assessments and the screening of the family members [18-20].

With the optimization of the materials and techniques, EUS is progressively assuming the prominent position as first choice imaging procedure for the assessment of the pancreas in MEN-1 patients, being superior to CT, MRI, and somatostatin receptor scintigraphy [21, 22]. In such patient population, somatostatin receptor scintigraphy is considered being the most reliable method for the detection of metastatic disease [4]. A possible limitation of EUS sensitivity for the detection of pNETs may be represented by the localization of the tumor, as the lesions localized in the tail of the pancreas might remain hidden, especially if they are small in size. The high sensitivity and high specificity of EUS for the detection of pNETs and its value for the ultrasonographic characterization of the tumor obviously depend very much on the experience and ability of the investigator. Furthermore, its cost effectiveness is an additional factor supporting the increasing use of this investigation [23]. Even though the availability of EUS has dramatically spread in the last decade and the technique is now very largely used in the third-level and many second-level hospitals, the unfortunate possibility of this investigation being not performed in the work-up of a suspicious pNET still exists and may depend on many factors.

In our study population, pNETs tend to be more numerous (almost twice) in patients with MEN-1 than in those with sporadic disease, which is a predictable obser- vation if we consider the pathogenesis of MEN-1-related versus sporadic lesions. This finding lacks statistical significance and can be affected by the size of the study population, which might not be large enough for confirming such observation also from a statistical point of view, and by the fact that our EUS study takes into consideration only the first investigation performed for each patient, thus ignoring the very likely subsequent detection of additional lesions overtime with the successive EUS assessments. However, once a pNET is disclosed at EUS for the first time in a MEN-1 patient, it appears to be probable that another or even more other pancreatic lesions are already detectable. On the other hand, sporadic pNETs are roughly 3 times bigger than those detected in MEN-1 patients and such a finding achieved a very strong statistical significance in our study population. While MEN1-related pNETs have usually a sub-centimeter diameter, sporadic pNETs often present with a diameter close to or bigger than $2 \mathrm{~cm}$.

With regard to the ultrasonographic features of the pNETs, only the heterogeneous versus the homogeneous appearance of the tumor seems to have a statistical relevance in the distinction between sporadic and MEN-1-related tumors. While sporadic pNETs are often heterogeneous, the lesions observed in MEN-1 patients are often homogeneous. To the best of our knowledge, a definite explanation for such EUS difference between the 2 patient groups is not available, though maybe the presence of areas of necrosis arising in the largest tumors might account for some intra-lesion ultrasonographic heterogeneity [24]. Interestingly and possibly somehow linked to this last observation, the majority of pNETs arising in MEN-1 patients have 
well-defined margins, while sporadic pNETs can equally present either with well-defined or not well-defined margins. However, the mild difference affecting the characteristics of the margins of the pNETs of the 2 patient groups did not achieve any statistical significance in our patient population, and maybe a comparison in other, or larger, patient populations might lead to a better definition of the clinical relevance of such EUS feature. Regardless of their pathogenesis, almost all pNETs have a round/oval shape and are hypervascular, highlighting a specific pattern that virtually characterizes the neuroendocrine neoplasms originating from Langerhans islet. We are of the opinion that a larger and multicenter patient population could elucidate further the EUS features of pNETs and we believe that a confirmation of our original data could increase the clinical relevance of this set of observations. Over and above the pNETs ultrasonographic characteristics observed through EUS, such a sensitive and specific investigation also represents a reliable and effective support for performing a lesion biopsy, with an obvious subsequent clinically relevant impact in terms of diagnostic characterization, pathogenetic definition, and prognosis of the pNET [25-27]. Moreover, EUS can also be associated to radiofrequency ablation of pancreatic tumors, including those of neuroendocrine origin [28].

In conclusion, EUS can help with the precise characterization of pNETs, including the definition of ultrasonographic features, which can distinguish MEN-1-related versus sporadic disease. At the time of the first EUS assessment of a patient with a pNET, the presence of a single big heterogeneous lesion suggests facing most likely a sporadic disease while the detection of 1 or more subcentimeter lesions with homogeneous aspect and welldefined margins is more likely to characterize a pNET related to MEN-1.

\section{Disclosure Statement}

The authors declare that they have no conflicts of interest to disclose.

\section{References}

1 Öberg K, Knigge U, Kwekkeboom D, Perren A; ESMO Guidelines Working Group: Neuroendocrine gastro-entero-pancreatic tumors: ESMO Clinical Practice Guidelines for diagnosis, treatment and followup. Ann Oncol 2012;23(suppl 7):vii124vii130.

2 Kondo NI, Ikeda Y: Practical management and treatment of pancreatic neuroendocrine tumors. Gland Surg 2014;3:276283.

3 Jensen RT, Berna MJ, Bingham DB, Norton JA: Inherited pancreatic endocrine tumor syndromes: advances in molecular pathogenesis, diagnosis, management, and controversies. Cancer 2008; $113(7$ suppl):1807-1843.

4 Thakker RV, Newey PJ, Walls GV, et al: Clinical practice guidelines for multiple endocrine neoplasia type 1 (MEN1). J Clin Endocrinol Metab 2012;97:2990-3011.

5 Triponez F, Dosseh D, Goudet P, et al: Epidemiology data on 108 MEN 1 patients from the GTE with isolated nonfunctioning tumors of the pancreas. Ann Surg 2006;243: 265-272.

6 Bartsch DK, Fendrich V, Langer P, Celik I, Kann PH, Rothmund M: Outcome of duodenopancreatic resections in patients with multiple endocrine neoplasia type 1. Ann Surg 2005;242:757-764

7 Triponez F, Goudet P, Dosseh D, et al: French Endocrine Tumor Study Group: Is surgery beneficial for MEN1 patients with small $(<$ or $=2 \mathrm{~cm}$ ), nonfunctioning pancreaticoduodenal endocrine tumor? An analysis of $65 \mathrm{pa}$ tients from the GTE. World J Surg 2006;30: 654-662.

8 Jensen RT, Cadiot G, Brandi ML, et al: ENETS Consensus Guidelines for the management of patients with digestive neuroendocrine neoplasms: functional pancreatic endocrine tumor syndromes. Neuroendocrinology 2012; 95:98-119.

9 Falconi M, Bartsch DK, Eriksson B, et al: ENETS Consensus Guidelines for the management of patients with digestive neuroendocrine neoplasms of the digestive system: well-differentiated pancreatic non-functioning tumors. Neuroendocrinology 2012;95:120-134.

10 Tamagno G, Sheahan K, Skehan SJ, et al: Initial impact of a systematic multidisciplinary approach on the management of patients with gastroenteropancreatic neuroendocrine tumor. Endocrine 2013;44:504-509.

11 Rösch T, Lightdale CJ, Botet JF, et al: Localization of pancreatic endocrine tumors by endoscopic ultrasonography. N Engl J Med 1992; 326:1721-1726.

12 Puli SR, Kalva N, Bechtold ML, et al: Diagnostic accuracy of endoscopic ultrasound in pancreatic neuroendocrine tumors: a systematic review and meta analysis. World J Gastroenterol 2013;19:3678-3684.

13 Langer P, Kann PH, Fendrich V, et al: Prospective evaluation of imaging procedures for the detection of pancreaticoduodenal endocrine tumors in patients with multiple endocrine neoplasia type 1 . World J Surg 2004;28: 1317-1322.

14 Thomas-Marques L, Murat A, Delemer B, et al: Prospective endoscopic ultrasonographic evaluation of the frequency of nonfunctioning pancreaticoduodenal endocrine tumors in patients with multiple endocrine neoplasia type 1. Am J Gastroenterol 2006;101:266273.

15 Kann PH, Balakina E, Ivan D, et al: Natural course of small, asymptomatic neuroendocrine pancreatic tumours in multiple endocrine neoplasia type 1: an endoscopic ultrasound imaging study. Endocr Relat Cancer 2006;13:1195-1202.

16 Collienne M, Timmesfeld N, Bergmann SR, Goebel J, Kann PH: Adrenal incidentaloma and subclinical Cushing's syndrome: a longitudinal follow-up study by endoscopic ultrasound. Ultraschall Med 2017;38:411-419.

17 Barnard GA: A new test for $2 \times 2$ tables. Nature 1945;156:177.

18 Tamagno G, De Carlo E, Martini C, Rubello D, Fallo F, Sicolo N: The early diagnosis of multiple endocrine neoplasia type 1 (MEN 1): a case report. J Endocrinol Invest 2004;27: 878-882.

19 Waldmann J, Fendrich V, Habbe N, et al Screening of patients with multiple endocrine neoplasia type 1 (MEN-1): a critical analysis of its value. World J Surg 2009;33:1208-1218. 
20 Sadowski SM, Triponez F: Management of pancreatic neuroendocrine tumors in patients with MEN 1. Gland Surg 2015;4:63-68.

21 Lewis MA, Thompson GB, Young WF Jr: Preoperative assessment of the pancreas in multiple endocrine neoplasia type 1. World J Surg 2012;36:1375-1381.

22 van Asselt SJ, Brouwers $\mathrm{AH}$, van Dullemen $\mathrm{HM}$, et al: EUS is superior for detection of pancreatic lesions compared with standard imaging in patients with multiple endocrine neoplasia type 1. Gastrointest Endosc 2015; 81:159-167.e2.

23 De Angelis C, Manfrè SF, Bruno M, Pellicano $\mathrm{R}$ : Hegemony and cost-effectiveness of endo- scopic ultrasound (EUS) in the field of gastroenteropancreatic-neuroendocrine tumors (GEP-NETs). Minerva Med 2014;105:363-370. 24 Dahan H, Soyer P, Cochand-Priollet B, et al: Imaging of primary carcinoid tumor of the pancreas. J Radiol 2001;82:987-990.

25 Chatzipantelis P, Salla C, Konstantinou P, Karoumpalis I, Sakellariou S, Doumani I: Endoscopic ultrasound-guided fine-needle aspiration cytology of pancreatic neuroendocrine tumors: a study of 48 cases. Cancer 2008; 114 : 255-262.

26 Chatzipantelis P, Konstantinou P, Kaklamanos M, Apostolou G, Salla C: The role of cytomorphology and proliferative activity in predicting biologic behavior of pancreatic neuroendocrine tumors: a study by endoscopic ultrasound-guided fine-needle aspiration cytology. Cancer 2009;117:211-216.

27 Reicher S, Boyar FZ, Albitar M, et al: Fluorescence in situ hybridization and K-ras analyses improve diagnostic yield of endoscopic ultrasound-guided fine-needle aspiration of solid pancreatic masses. Pancreas 2011;40:10571062.

28 Pai M, Habib N, Senturk H, et al: Endoscopic ultrasound guided radiofrequency ablation, for pancreatic cystic neoplasms and neuroendocrine tumors. World J Gastrointest Surg 2015;7:52-59.

\section{Free Author Copy - for personal use only}

ANY DISTRIBUTION OF THIS ARTICLE WITHOUT WRITTEN CONSENT FROM S. KARGER AG, BASEL IS A VIOLATION OF THE COPYRIGHT. Written permission to distribute the PDF will be granted against payment of a permission fee, which is based on the number of accesses required. Please contact permission@karger.com 